\title{
Large eddy simulation of turbulent combustion in a spark assisted homogenous charge compression ignition engine
}

\author{
T. Joelsson, R. Yu and X.S. Bai \\ Xue-Song.Bai@energy.lth.se \\ Division of Fluid Mechanics, Lund University, 22100 Lund, Sweden
}

\begin{abstract}
A large eddy simulation (LES) model was developed to simulate the combustion process in a spark-assisted homogeneous charge compression ignition (SACI) engine. First, an ignition and flame propagation model based on a reaction progress variable is presented. The reaction progress variable is defined based on the normalized cumulative heat release. Transport equation for the progress variable is derived where the source terms due to flame propagation and auto-ignition are modelled. The model is then applied to simulate the SACI combustion process with special focus on the interaction between the flame propagation introduced by the spark and the auto-ignition of the homogeneous charge. The engine simulated is a 0.5 litre experimental HCCI engine, with operation conditions ranging from spark-ignition controlled flame propagation to auto-ignition controlled HCCI combustion. In the first stage of SACI combustion, between the spark-ignition and the onset of HCCI auto-ignition, turbulence field governs the heat release rate and pressure-rise-rate in the cylinder. Increasing turbulence promotes the contribution of SI flame to the overall heat release. The second stage combustion, which is in the HCCI auto-ignition mode, is rather sensitive to the temperature field. The numerical results showed that with low initial temperature the SI flame mode prevails; with high initial temperature the HCCI mode prevails. With moderate initial temperature SI flame and HCCI ignition interact more closely, which results in higher sensitivity to the initial temperature and turbulence conditions. This may be the reason of having high cyclic variation found in the previous experiments.
\end{abstract}

\section{Introduction}

In light of today's public concern on green house gas $\left(\mathrm{CO}_{2}\right)$ emission and air pollution from combustion of fossil fuels, modern internal combustion engines are developed to have high efficient with low emissions. The benefits and shortfalls of the two major combustion concepts, spark ignition (SI) and compression ignition (CI), have over the past decades led to the development of homogenous charge compression ignition (HCCI) engines that can achieve high efficiency and low emissions of NOx and soot by using high compression ratio and excessive air or exhaust gas recirculation (EGR) in the fuel/air mixtures [1-4].

In HCCI engines the combustion phasing is controlled by the auto-ignition of the lean charge. As the ignition delay time is sensitive to temperature of the charge the combustion phasing becomes rather sensitive to the initial flow and intake flow conditions. Furthermore, in HCCI engines the reaction fronts propagate at a velocity typically of an order of magnitude higher than the turbulent flame speed, the combustion duration in HCCI engine can be short if care is not taken to generate a suitable flow and temperature field in the cylinder. This is especially a serious problem when the engine runs at high load, where the pressure-rise-rate can be rather high, resulting in high noise level [5]. A recent review on HCCI combustion can be found in Yao et al. [6].

One way to control the ignition timing (combustion phasing) in HCCI engines is to ignite the fuel/air mixture with a spark before the onset of auto-ignition [7-10]. This strategy is known as spark-assisted HCCI (SACI), which may also be viewed as a natural extension of 
the gasoline SI engine operation in a HCCI mode at low load by trapping hot residual gas (internal EGR) or external EGR. In SACI a flame kernel is first initiated, followed by autoignition of the remaining charge. A successful SACI operation would depend on the success in manipulation of the SI flame/auto-ignition interaction.

Several experimental studies have been conducted to investigate how the engine operation conditions, e.g. spark timing, load and amount of EGR in the mixture [11], swirl and thereby level of turbulence [12], fuel stratification by using secondary direct injection [13], on SACI combustion. Important information has been obtained in these investigations; for example, by using inlet valve deactivation to increase swirl and thus the level of turbulence, the SI flame contribution to the overall heat release is increased and the HCCI auto-ignition process is delayed at high-level EGR conditions [12]. It is fairly well recognized that turbulence can directly interact with the premixed flame propagation initiated by the spark, e.g. by wrinkling the flame fronts; however, the effect of turbulence on HCCI combustion can be rather problem dependent. It is generally accepted that temperature stratification plays an important role in HCCI combustion [14-16]. With large temperature stratification the combustion duration can be longer and the pressure-rise-rate can be lower for a given combustion phasing. One important role of turbulence played in HCCI engines is by modulating the temperature stratification in the engine cylinder; turbulence can affect the mixing of the intake gas with the residual gas and similarly it can affect the heat transfer between the intake gas and the hot residual gas, and between the cylinder/piston walls and the charge $[17,18]$. Under certain conditions, e.g. low intensity turbulence and high temperature stratification [19], or when the integral scale of turbulence eddies are comparable with the length scales of the hot/cold spots [16], turbulence can directly interact with the ignition front.

It is evident that the interaction between the SI flame and the HCCI combustion in a SACI engine can be highly nonlinear and under certain EGR level the EGR nonlinear feedback mechanism can lead to oscillatory combustion and cyclic variation [20]. It is yet unclear how the two processes interact each other under different initial mixture and engine operation conditions. The goal of this work is to gain more insights to interaction between the SI flames and the HCCI auto-ignition fronts. A SACI model based on large eddy simulation (LES) approach is presented in this paper; the model is used to simulate an experimental SACI engine [13] where incylinder pressure measurement was reported. The transition region between SI flame and HCCI combustion is simulated.

\section{Description of the SACI LES model}

A LES model is developed for SACI combustion. The model is based on a reaction progress variable that is defined based on the normalized cumulative heat release [21]. The SI premixed flame propagation model is based on the flame surface density concept [22-24]. Spatially filtered Navier-Stokes equations and energy transport equations are coupled with the progress variable equation. Inside the SI premixed flame kernels the combustion products are computed using tabulated flamelet database [25,26]; outside the SI flame kernels the species and temperature are computed using an ignition tabulation database based on enthalpy, pressure and the ignition progress variable [19]. The SI flame and the HCCI ignition process interact through the incylinder pressure, temperature, as well as the heat and mass transfer by turbulence between the SI flame kernels and the unburned charge.

The reaction progress variable for SACI combustion

First, we introduce a reaction progress variable defined as the following $[21,19]$,

$$
c=\frac{h\left(T_{r e f}, Y_{i}\right)-h\left(T_{r e f}, Y_{i, u}\right)}{h\left(T_{r e f}, Y_{i, b}\right)-h\left(T_{r e f}, Y_{i, u}\right)}
$$


where $h\left(T_{r e f}, Y_{i}\right)$ is the specific enthalpy of the charge defined at the reference temperature $T_{\text {ref }}$ of $298 \mathrm{~K} ; Y_{i, u}$ is the mass fraction of species $i$ in the unburned charge; $Y_{i, b}$ is mass fraction of species $i$ in the fully burned charge. It appears that

$$
h\left(T_{\text {ref }}=298 K, Y_{i}\right)=\sum_{i=1}^{N} Y_{i} h_{i, f}^{0}
$$

where $h_{i, f}^{0}$ is enthalpy of formation at standard (reference) condition. $h\left(T_{\text {ref }}, Y_{i}\right)$ represents the heat release in the combustion process, whereas $c=0$ corresponds to the state of unburned and $c=1$ corresponds to the end state of combustion that all heat has been released. $c$ can therefore be interpretated as the normalized cumulative heat release. A transport equation for $c$ can be derived from the conservation of mass and equations for the species transport by assuming the Lewis numbers are unity:

$$
\frac{\partial \rho c}{\partial t}+\nabla \cdot(\rho \vec{v} c)=\nabla \cdot(\rho D \nabla c)+\dot{\omega}_{c}
$$

where $\rho$ is density; $\vec{v}$ is velocity vector; $D$ is mass diffusion coefficient. The source term $\dot{\omega}_{c}$ is obtained from models described below. In the LES context the above equation and other transport equations (e.g. for mass, momentum and energy) are spatially filtered, which results in unknown terms in the transport equations; these terms represent the effect of subgrid scale (SGS) on the resolved scale. In the present numerical solver $[16,17,19]$ the SGS fluxes in the transport equations for scalars (the specific enthalpy, and the progress variable) are modelled using the Smagorinsky model [27], whereas the SGS stresses in the momentum equations are modelled using scale-similarity model [28].

Models for HCCI front propagation

For HCCI combustion in a homogeneous composition (but not homogeneous in temperature) field, the source term $\dot{\omega}_{c}$ is obtained from numerical calculations (with detailed chemical kinetic mechanisms) of the ignition process in a homogenous mixture with a given initial enthalpy (or temperature) and pressure. From the numerical simulations the species mass fractions, temperature and the progress variable are tabulated as a function of time. The ignition calculations are performed for a range of initial enthalpy and pressure, based on which the rate of change of $c$ (the source term in Eq.3) can be tabulated as a function of the initial pressure $(p)$, enthalpy and the progress variable itself,

$$
\dot{\omega}_{c}=\frac{d c}{d t}=f_{0}(h, p, c)
$$

In engine calculations, when $c, h$, and $p$ are known, temperature of the charge at each grid point can be computed from such auto-ignition tabulation. Thereafter the thermodynamic pressure (incylinder pressure) can be determined from global mass conservation and the equation of state. Local density of the charge is determined from the equation of state. In the LES context, the SGS effect on the source term has to be considered through presumed probability density function (PDF) approach [19].

\section{Models for SI premixed flame propagation}

There are several different types of models developed for premixed flame propagation; examples are G-equation based level-set approach [25], reaction-rate-based progress variable approach [22-24], and PDF statistical approaches [29], etc. These approaches are interconnected as they model the same physical process. In this work, we adopt the rate-based 
progress variable approach to be consistent with the HCCI ignition model discussed above. This facilitates a consistent and easier implementation of the two models.

We adopt the same definition of progress variable $c$ as in Eq.(1). Namely, for the SI premixed flame $c$ is also the normalized cumulative heat release. Thus, transport equation for $c$ is identical to Eq.(3). Different from the HCCI model, the reaction rate for SI premixed flame propagation in the LES context is modeled as follows,

$$
\dot{\omega}_{c}=\rho_{u} s_{L} \sum
$$

where $\rho_{u}$ is the density of the unburned charge, $s_{L}$ is the laminar burning velocity of the charge, and $\sum$ is the flame surface density. In [22] a transport equation for $\sum$ was proposed. There are several unknown closure terms needs to be modeled. In LES when the spatial filter size is small, we may use a simplified model for $\sum$ as derived below. First, from Eq.(3) it can be shown that

$$
\int_{-\infty}^{+\infty} \dot{\omega}_{c} d x=\llbracket \rho v_{n} c \rrbracket+\int_{-\infty}^{+\infty} \frac{\partial \rho c}{\partial t} d x=\llbracket \rho v_{n} c \rrbracket \equiv \dot{m} \equiv \rho_{u} s_{s g s}
$$

where $\dot{m}$ is the mass flux burned by the flame; $s_{s g s}$ is the burning velocity on the resolved LES scale. Since $\sum$ is likely high in the middle of the flame brush and small as at the edge of the flame one can assume that

$$
\sum=A(1-c) c
$$

where A is a proportionality constant to be determined. From Eqs.(5,6,7) it appears that

$$
\rho_{u} s_{s g s}=\int_{-\infty}^{+\infty} \dot{\omega}_{c} d x=\rho_{u} s_{L} \int_{-\infty}^{+\infty} \sum d x=\rho_{u} s_{L} A \int_{-\infty}^{+\infty}(1-c) c d x=\rho_{u} s_{L} A g
$$

Thus,

$$
A g=s_{s g s} / s_{L} \simeq 1+u^{\prime}
$$

where $g$ is an integral to be discussed further in Eq.(11). In Eq.(9) $s_{s g s}$ was modeled using Damköhler's turbulent flame speed model, which is valid in the flamelet regime of turbulent premixed flames. $u^{\prime}$ is the SGS velocity estimated using Smagorinsky model based on the resolved scale flow strain rate [26]. Naturally, other models can be used if the flames are not in the flamelet regime. From Eqs.(5-9) one has

$$
\begin{aligned}
\dot{\omega}_{c} & =\rho_{u} s_{L} \sum=\rho_{u} s_{L} A(1-c) c=\rho_{u} s_{s g s} g^{-1}(1-c) c \\
& =\rho_{u} s_{L} g^{-1}\left(1+u^{\prime}\right)(1-c) c \\
& =\rho_{u} s_{L}\left(1+u^{\prime}\right)(1-c) c\left(\int_{-\infty}^{+\infty}(1-c) c d x\right)^{-1}
\end{aligned}
$$

$g$ can be estimated as follows,

$$
g=\int_{-\infty}^{+\infty}(1-c) c d x \simeq\left(\frac{d c}{d x}\right)^{-1} \int_{0}^{1}(1-c) c d c=\left(\frac{d c}{d x}\right)^{-1} / 6 \simeq \alpha \Delta / 6
$$

where $\Delta$ is the LES filter size. In Eq.(11) the mean gradient of the reaction progress variable has been estimated as $1 / \alpha \Delta$, by assuming that filtered reaction zone has a thickness of $\alpha \Delta$. The model parameter $\alpha$ represents the ratio of the thickness of the filtered reaction zone to 
the filter size. In this study, the filter size was set to be the grid size, and $\alpha$ was set to 6 , which implies that the reaction zone is filtered to within 6 grid cells. This is found to be helpful for the stability of the numerical solver. From Eqs.(10,11),

$$
\dot{\omega}_{c}=\frac{6 \rho_{u} s_{L}\left(1+u^{\prime}\right)(1-c) c}{\alpha \Delta}
$$

Once $c$ is computed, we can also use the flamelet library approach to determine local species, temperature and then pressure as well as density [26]. In the current case, the flamelet library is equivalent to the tabulation of the HCCI auto-ignition library.

Coupling of the SI premixed flame model and the HCCI model

In SACI engine simulations, the two models can be coupled as follows,

$$
\dot{\omega}_{c}=\max \left(\dot{\omega}_{c, A I}, \dot{\omega}_{c, P F}\right)
$$

In places where HCCI ignition is important the rate from the HCCI model will be higher than that from the premixed flame propagation. In places where temperature is too low to have auto-ignition, the rate from the HCCI model will be low; the premixed flame will be dominant. In places where it is possible to have both HCCI and premixed flame, the dominant modes would have higher rates. Thus, Eq.(13) is a reasonable model.

Alternatively, one can employ two progress variables (and thus two transport equations for the progress variables) to couple the SI flame and HCCI ignition; one progress variable is to track the SI flame propagation, and one for the HCCI ignition process. The coupling is more straightforward: at a given spatial location in the cylinder when the progress variable for the SI flame is higher than that for the HCCI ignition, it implies that the SI flame prevails in the given location. As such one can use the maximum of the two progress variables to determine the thermodynamic variables. This approach is used in the following study.

\section{Engine setup and computational conditions}

The engine studied here is an experimental HCCI engine with a displacement volume in a cylinder of $480 \mathrm{~cm}^{3}$ [13]. The engine ran at $1200 \mathrm{rpm}$ with a compression ratio of 12 . The engine has a bore of $81 \mathrm{~mm}$, and a stroke of $93.2 \mathrm{~mm}$. The fuel is ethanol with the equivalence ratio of 0.61 , supplied to the cylinder through port-fuel injection, which allows for the fuel and air to mix well in the cylinder. Hot residual gas was trapped in the cylinder by negative valve overlap (NVO) strategy; the mass fraction of the residual gas in the cylinder after the intake valve close is about 0.3. Numerical study of the mixing process showed that there is moderate inhomogeneity in the mixture composition due to the later NVO valve timing. It was found that the ignition process is more sensitive to the temperature stratification than to the composition stratification in the present case. For simplicity, the stratification in the composition is neglected here.

In Table 1 nine different test cases are listed, including three HCCI cases and six SACI cases with different in-cylinder temperature and turbulence conditions. The mean in-cylinder gas temperature, the fluctuations of the temperature, and the level of turbulence at $290 \mathrm{CAD}$ were varied to investigate the sensitivity of the combustion behaviour to these parameters, especially the onset of auto-ignition of the mixture and its interaction with the SI flame propagation under different temperature stratification and turbulence conditions. The initial instantaneous flow and thermodynamic variables at 290 CAD were generated from LES of the intake and compression stroke starting from the intake TDC (0 CAD), where the initial gas (which is the residual gas trapped from the previous cycle) temperature is set to $662 \mathrm{~K}$, based on the engine experimental data [13]. The instantaneous velocity field at 290 CAD is spatially filtered using a Gaussian filter function with a filter size of half the bore; the rms 
velocity associated with the filtered small-scale flow structures is then volume averaged based on the entire cylinder (denoted in Table 1 as $u_{r m s}^{\prime}$ ). It represents the level of turbulence in the entire cylinder. From the LES result, $u_{r m s}^{\prime}$ is $3.1 \mathrm{~m} / \mathrm{s}$. In Table $1, T_{r m s}^{\prime}$ is the rms temperature computed based on the instantaneous and the cylinder volume averaged mean temperature at 290 CAD. The different turbulence and temperature conditions at 290 CAD shown in Table 1 are implemented by scaling the instantaneous velocity and temperature fields.

Table 1. Simulation cases and initial conditions at CAD 290. For the SACI cases the sparkignition starts at $320 \mathrm{CAD}$. Units: $\langle T\rangle$ and $T_{r m s}^{\prime}$ in $\mathrm{K} ; u_{r m s}^{\prime}$ in $\mathrm{m} / \mathrm{s}$.

\begin{tabular}{|c|c|c|c|c|c|c|c|c|c|}
\hline Cases & Saci-1 & Saci-2 & Saci-3 & Saci-4 & Saci-5 & Saci-6 & Hcci-1 & Hcci-2 & Hcci-3 \\
\hline$\langle T\rangle$ & 580 & 620 & 620 & 650 & 670 & 650 & 620 & 650 & 670 \\
\hline$T_{r m s}^{\prime}$ & 50 & 20 & 50 & 20 & 20 & 20 & 20 & 20 & 20 \\
\hline$u_{r m s}^{\prime}$ & 3.1 & 3.1 & 3.1 & 3.1 & 3.1 & 0.5 & 3.1 & 3.1 & 3.1 \\
\hline
\end{tabular}

In the SACI cases the spark is modelled as a spherical flame kernel with the diameter of $3.8 \mathrm{~mm}$ within which the reaction progress variable was set to 1 . The spark ignition time in all SACI cases is set at 320 CAD, i.e. 40 CAD before TDC.

The simulations were performed using an inhouse LES code $[16,17,19]$. The code is based on a high order numerical discretization scheme (fourth order central difference/fifth order WENO) on a deforming Cartesian grid to accommodate the piston motion. The code has been validated in several engine configurations with satisfactory results $[17,19]$. In the present simulations the grid used is $128 \times 128 \times 128$. With 8 processors the simulation took about 24 CPU hours per engine cycle.

\section{Results and discussions}

Figure 1 shows the development of SI flame front (the dark solid line) and the instantaneous temperature field at different crank angles for the SACI-2 case. At 324 CAD, i.e., 4 CAD after the flame kernel was initiated in the middle of the cylinder, the flame kernel is distorted from its initial spherical shape. The size of the kernel is still rather small. The flame kernel is not significantly larger than the resolved turbulence eddies so that the flame surface is not wrinkled. At $332 \mathrm{CAD}$, i.e. $12 \mathrm{CAD}$ after the start of ignition, the flame kernel has grown larger and the flame surface shows wrinkling. From 332 CAD to 359 CAD the SI premixed flame propagates from the central ignition site to become highly wrinkled large flame. As the premixed flame propagates and the piston moves to its TDC position the incylinder pressure increases due to compression and heat release, which results in an increase in the temperature of the unburned charge outside the flame. At 359 CAD there is no significant auto-ignition kernel seen in the charge in the shown cross section; however, 3 CAD later, at 362 CAD the charge in multiple sites outside the flame kernel become auto-ignited. 2 CAD later, at 364 CAD, most of the charge outside the flame kernel become ignited due to rapid HCCI autoignition. The premixed flame front is seen to propagate to the burned region after $362 \mathrm{CAD}$, which has no direct physical meaning, but rather it is used to demonstrate the relative speed of ignition front propagation and flame front propagation. 


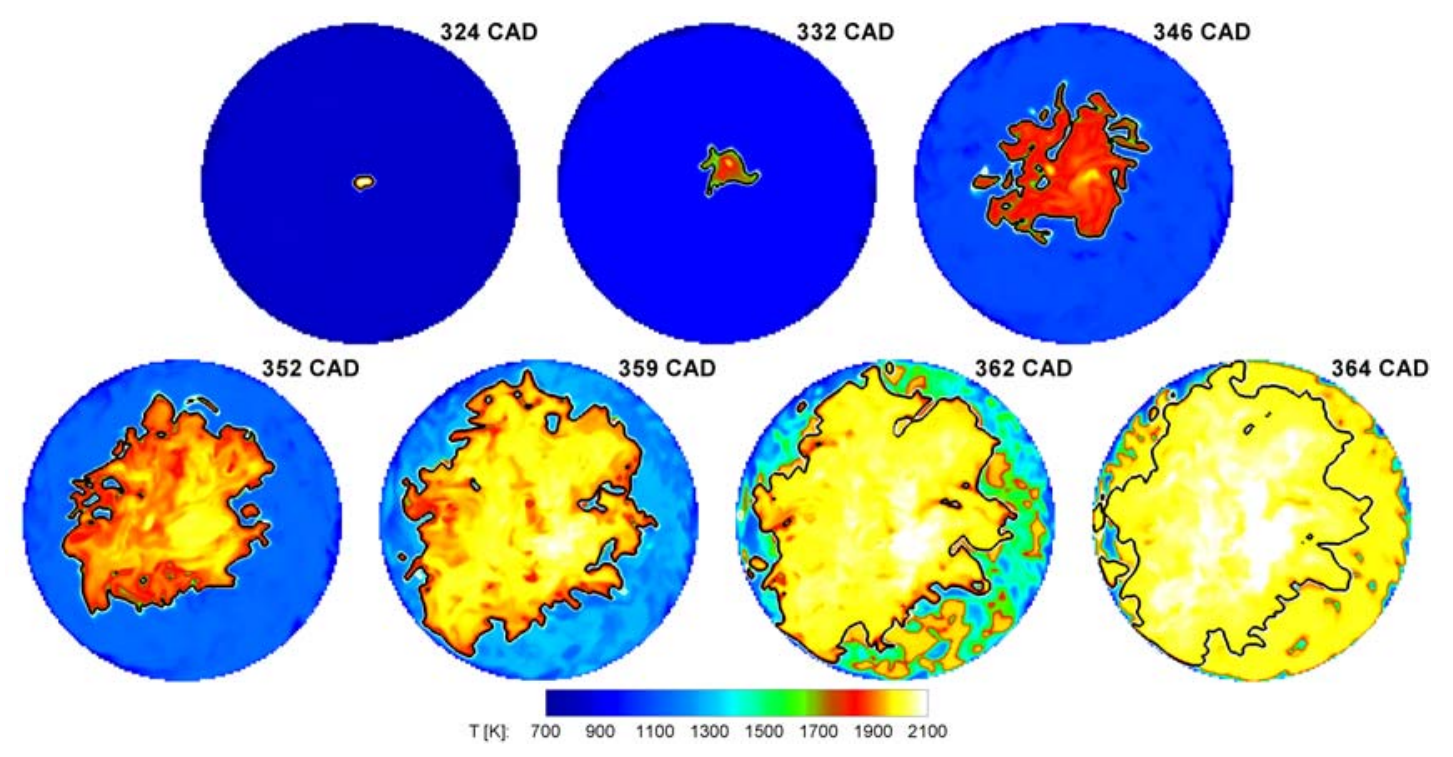

Figure 1. Instantaneous temperature field in a cross section of the cylinder for case SACI-2.

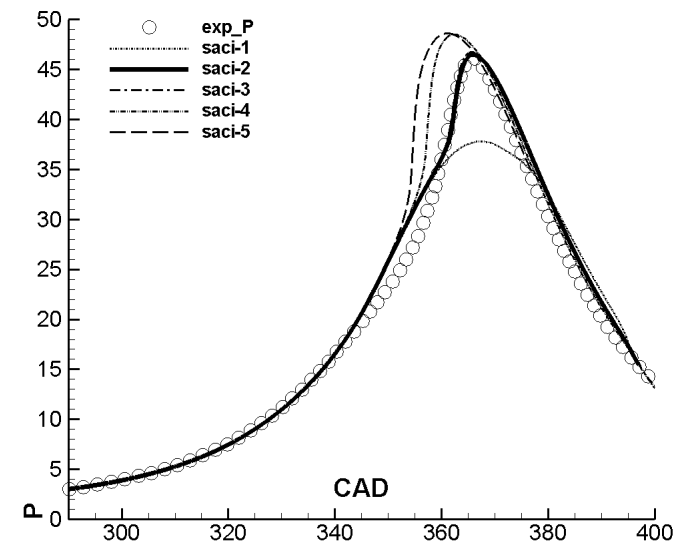

Figure 2. Incylinder pressure under different SACI and HCCI conditions.

The incylinder pressure for case SACI-2 is shown in Fig.2; the result is comparable with the experimental result. Also shown in the figure are the results of other SACI cases. These results reveal the effect of temperature field on the SACI combustion process. For case SACI1 with lower initial temperature of 580K, HCCI auto-ignition is by-passed, yielding a much lower incylinder pressure peak as compared with the result of SACI-2 and the experiments. For SACI-3 the initial mean temperature is identical to SACI-2 but the stratification of temperature in SACI-3 is higher than that in SACI-2. The two cases have identical incylinder pressure (in the figure they overlap each other). This shows that temperature stratification played minor role in the present case, which is due to the fact that in SACI-2 and SACI-3 the HCCI duration is very short, ranging from 359 CAD to 364 CAD, such that the effect of temperature stratification would not lead to significant difference in the ignition delay time in the charge. For SACI-4 and SACI-5 the initial mean temperature was increased and as such the onset of HCCI ignition becomes earlier, the pressure-rise-rate becomes higher, and the contribution from HCCI ignition to the overall heat release becomes higher.

The effect of SI flame propagation on the overall combustion process can be further examined by comparing the three HCCI cases (shown in Fig.3) with the SACI cases (Fig.2). 
HCCI-1 with the same initial condition as SACI-2 has no spark ignition. Figure 3 shows that HCCI-1 failed to ignite. HCCI-2 has the same initial condition as SACI-4. One can see that without the spark ignition, HCCI-2 is ignited later with the peak pressure much lower than the experiment case. HCCI-3 has the same initial condition as SACI-5. Without the SI flame HCCI-3 was shown to auto-ignite at TDC, also much later than case SACI-5.

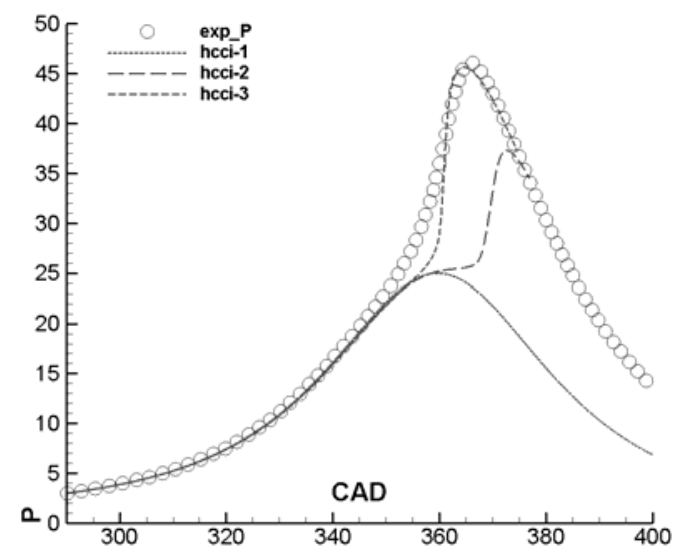

Figure 3. Mean temperature in the cylinder under different SACI and HCCI conditions.

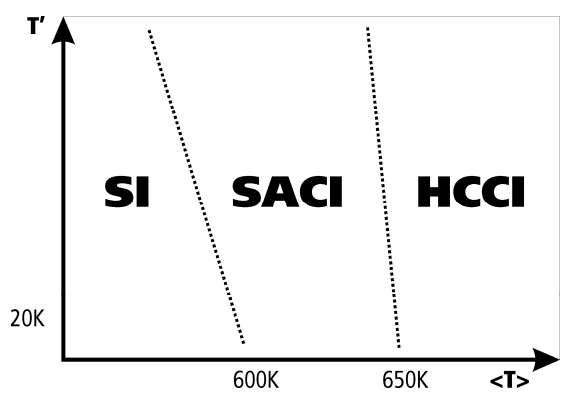

Figure 4. Schematic illustration showing the dependence of SI, SACI and HCCI combustion on the mean temperature and temperature stratification.

Figure 4 summarizes the effect of initial temperature field on SACI combustion. When the initial temperature is low, e.g. SACI-1, the main contribution to heat release and combustion is from the SI flame propagation. Auto-ignition would not occur with low initial temperature since the heat release rate from SI flame propagation is relative slow, which would not increase the temperature in the charge fast enough before the expansion stroke starts. This situation is somewhat similar to the SI engine operating at low load (in Fig.4, it corresponds to the SI-regime). When the initial temperature is significantly increased, e.g. SACI-5 and HCCI-3, auto-ignition would occur with or without the SI flame. It can be expected that further increase the initial temperature the contribution to combustion/heat release from SI flame would decrease, and the dependence of the engine performance on the spark ignition would be minor. This is the HCCI-regime in Figure 4; it is similar to the situation of gasoline HCCI with high EGR. When the initial temperature is moderately high, e.g. SACI-2, SACI-3, SACI-4, HCCI-1, and HCCI-2, the SI flame and HCCI auto-ignition interact more closely. Removing the spark ignition the engine may change from stable operation to misfire, e.g. HCCI-1/SACI-2; or may change to partially burn, e.g. HCCI-2/SACI-4. This indicates a high sensitivity of the combustion process to the initial temperature field. This situation is similar 
to gasoline SI engine with moderate EGR, which has shown oscillatory combustion and highlevel cyclic variation [20].

Figure 5 shows the effect of turbulence on the SACI combustion process. In SACI-4 and SACI-6 the initial turbulence rms velocities are different, while other conditions are identical. It is seen that when turbulence velocity is decreased the pressure-rise-rate in the SI flame propagation stage decreases, which leads to a later HCCI auto-ignition. The slower pressure increase is a result of slower SI flame propagation, due to the lower degree of flame front wrinkling.

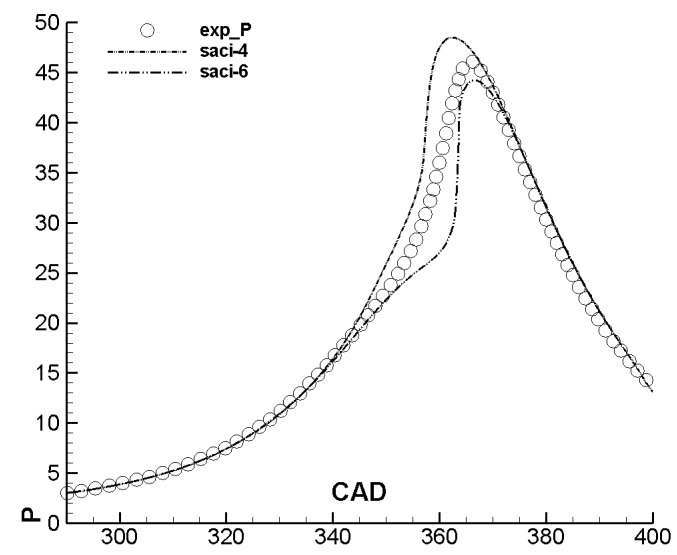

Figure 5. Pressure and mean temperature in the cylinder under different SACI and turbulence conditions.

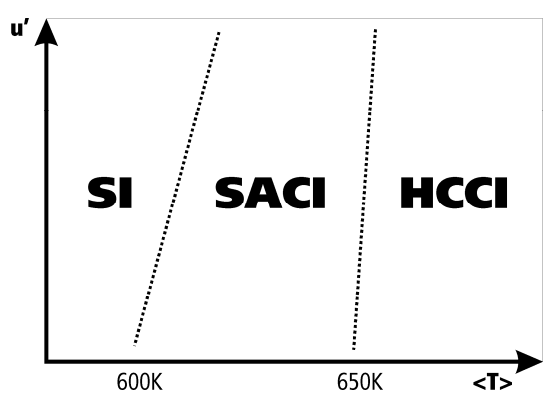

Figure 6. Schematic illustration showing the dependence of SI, SACI and HCCI combustion on the mean temperature and temperature stratification.

In Fig.6 a summary of the effect of turbulence on SACI combustion is presented. At low initial temperature conditions, since SI flame propagation will prevail, increasing turbulence would enhance the flame wrinkling and flame propagation. Thus the SI-regime would be increased. At high initial temperature conditions, since HCCI mode will be dominant, increasing turbulence would modify the temperature field and as such the ignition front propagation will be affected. However, it is expected that effect of turbulence on the HCCIregime will be less significant than on the SI-regime. Under moderate initial temperature conditions, turbulence would play a significant role. Turbulence would increase the speed of heat release from SI flame propagation, and as such it promotes the onset of HCCI ignition, e.g. SACI-4/SACI-6. 


\section{Conclusions}

LES of a personal car sized experimental SACI engine is performed to analyze the effect of initial temperature and turbulence fields on the SACI process. SACI combustion can be divided to two stages, one initial SI flame stage followed by the HCCI auto-ignition stage. The second stage is often very fast as indicated by the much rapid pressure-rise-rate as compared with the SI flame propagation stage. A LES SACI model is presented, which is based on the normalized cumulative heat release as a reaction progress variable. The LES results show that the SACI operation window can be rather narrow: with too low initial temperature (for example controlled by inlet temperature) the second stage HCCI combustion can be by-passed, yielding a semi-misfire operation. On the other hand, if the initial temperature is too high the SI flame may not be effective. It is seen that turbulence plays significant role in the first stage SI flame propagation, whereas initial temperature governs the second stage HCCI process.

\section{Acknowledgements}

The authors gratefully acknowledge the Swedish Energy Agency (STEM), the Swedish Research Council (VR) and the Competence Centre Combustion Processes (KCFP) at Lund University for their financial support, and Lunarc for the computer resources.

\section{References}

[1] S. Onishi, J. Hong, K. Shoda, P.J. Do, S. Kato, Active thermoatmosphere combustion ATAC - A new combustion process for internal combustion engines, SAE paper 19790507.

[2] M. Christensen, B. Johansson, Influence of Mixture Quality on Homogeneous Charge Compression Ignition, SAE paper 1998-2454.

[3] P.M. Najt, D.E. Foster, Compression-Ignited Homogeneous Charge Combustion, SAE paper 1983-0264.

[4] D.N. Assanis, P.M. Najt, J.E. Dec, J.A. Eng, T.N. Asmus, F. Zhao, Homogeneous Charge Compression Ignition (HCCI) Engines, SAE International, 2003.

[5] J.O. Olsson, P. Tunestål, B. Johansson, Boosting for high load HCCI, SAE paper 200401-0940.

[6] M. Yao, Z. Zheng, H. Liu, Progress and recent trends in homogeneous charge compression ignition (HCCI) engines, Progress in Energy and Combustion Science 35 (2009) 398-437.

[7] L. Koopmans, I. Denbratt, A four stroke camless engine, operated inhomogeneous charge compression ignition mode with commercial gasoline, SAE paper 2001-01-3610.

[8] A. Fuerhapter, W.F. Piock, G.K. Fraidl, CSI - controlled auto-ignition - the best solution for the fuel consumption - versus emission trade-off, SAE paper 2003-01-0754.

[9] H. Persson, R. Pfeiffer, A. Hultqvist, B. Johansson, H. Ström, Cylinder-to-cylinder and cycle-to-cycle variations at HCCI operation with trapped residuals, SAE paper 2005-010130 .

[10] T. Urushihara, K. Yamaguchi, K. Yoshizawa, T. Itoh, A study of a gasoline-fueled compression ignition engine - extension of HCCI operation range using SI combustion as a trigger of compression ignition, SAE paper 2005-01-0180.

[11] H. Persson, A. Hultqvist, B. Johansson, A. Remon, Investigation of the earlier flame development in spark-assisted HCCI combustion using high speed chemiluminescence imaging, SAE paper 2007-01-0212.

[12] H. Persson, B. Johansson, A. Remon, The effect of swirl on spark assisted compression ignition (SACI), SAE paper 2007-01-1856. 
[13] H. Persson, J. Sjöholm, E. Kristensson, B. Johansson, M. Richter, M. Aldén, Study of Fuel Stratification on Spark Assisted Compression Ignition (SACI) Combustion with Ethanol Using High Speed Fuel PLIF, SAE paper 2008-01-2401.

[14] J. Ozaki, N. Iida, Efeect of degree of unmixedness on HCCI combustion based on experiment and numerical analysis, SAE Paper 2006-32-0046.

[15] M. Sjöberg, J.E. Dec, N.P. Cernansky, Potential of thermal stratification and combustion retard for reducing pressure-rise rates in HCCI engines, based on multi-zone modeling and experiments, SAE paper 2005-01-0113.

[16] R. Yu, T. Joelsson, X.S. Bai, B. Johansson, Effect of Temperature Stratification on the Auto-ignition of Lean Ethanol/Air Mixture in HCCI engine, SAE paper 2008-01-1669.

[17] R. Yu, X.S. Bai, A. Vressner, A. Hultqvist, B. Johansson, J. Olofsson, H. Seyfried, J. Sjöholm, M. Richter, M. Aldén, Effect of Turbulence on HCCI Combustion, SAE 200701-0183.

[18] S.C. Kong, R.D. Reitz, M. Christensen, B. Johansson, Modeling the Effects of Geometry Generated Turbulence on HCCI Engine Combustion, SAE 2003-01- 1088.

[19] R. Yu, X.S. Bai, H. Lehtiniemi, S.S. Ahmed, F. Mauss, M. Richter, M. Aldén, L. Hildingsson, B. Johansson, A. Hultqvist, Effect of Turbulence and Initial Temperature Inhomogeneity on Homogeneous Charge Compression Ignition Combustion, SAE 200601-3318.

[20] C.S. Daw, R.M. Wagner, K. D. Edwards, J.B. Green, Understanding the transition between conventional spark-ignited combustion and HCCI in a gasoline engine, Proc. Combust. Inst. 31 (2007) 2887-2894.

[21] Y. Zhang, B. Rogg, K.N.C. Bray, 2-D Simulation of Turbulent Autoignition with Transient Laminar Flamelet Source Term Closure, Combustion Science and Technology Vol. 105 pp. 211-227 (1995).

[22] S. M. Candel, T. Poinsot. Flame stretch and the balance equation for the flame surface area. Combust. Sci. Tech., 70:1-15, 1990.

[23] R.S. Cant, K.N.C.Bray, A theoretical model of premixed turbulent combustion in closed vessels, Combustion and Flames, Vol. 76, pp. 243-263 (1989).

[24] G.M. Abu-Orf, R.S. Cant, A turbulent reaction rate model for premixed turbulent combustion in spark-ignition engines, Combustion and Flames, Vol.122, pp.233-252 (2000).

[25] N. Peters. Turbulent combustion. Cambridge University Press, 2000.

[26] P. Wang, X.S. Bai. Large eddy simulation of turbulent premixed flames using level-set G-equation. Proc. Combust. Inst., 30:583-591, 2005.

[27] J. Smagorinsky. General circulation experiments with the primitive equations. Monthly weather Review, 91:99-152, 1963.

[28] S. Liu, C. Meneveau, J. Katz. On the properties of similarity subgrid-scale models as deduced from measurements in a turbulent jet, J. Fluid Mech., 275:83-119, 1994.

[29] S.B. Pope. Pdf methods for turbulent reactive flows. Prog. Energy Comb. Sci., 19:119192, 1985. 